\title{
Epithelial excrescence, protuberance, hypertrophy: the oral cicatrix
}

\section{Introduction}

A benign proliferation of the stratified squamous epithelium terminating in a papillary or verrucous exophytic conglomeration is designated as an Oral Squamous Papilloma (OSP). ${ }^{1}$ The tongue and soft palate are common locations, however several oral surfaces may be implicated. ${ }^{1}$ With Human Papilloma Virus (HPV) as a frequent contagion, a 150 varieties of virus have been indicated in the genesis of the lesion. ${ }^{1}$ Malignant transformation is potentially associated with the HPV's 16, 18, 31, 33, 35, 39, 45, 51, 55, 56, 58, 59, 66 and $68 .^{1,2}$ The viruses engender the evolution of diverse human neoplasm, particularly the epithelium of the uterine cervix, urethra, ano-genital region, skin, digestive tract, trachea/bronchi, nasal mucosa and oral mucosa. ${ }^{1-3}$

Preface: Oral Squamous Papilloma occurs preponderantly in females with a variable age of presentation. Specific clinical and physical attributes may be observed. Excision biopsy, histopathology and congruous immunohistochemical parameters may be appraised. Invasive squamous cell carcinoma with the progenitors such as cervical intraepithelial neoplasm (grade III) and carcinoma in situ may incorporate the nuclear (DNA) sequences of HPV 16 and 18 in numerous instances. ${ }^{4-6}$ Human Papilloma Virus contamination activates the benign cellular expanse. Subsequent somatic mutations may assist the propagation of the virus. Pathogenic determinants of malignant conversion such as smoking, concomitant infections, dietary deficiencies, hormonal changes may expedite the cellular adaptations. The specific HPV-DNA may be amply evidenced in the neoplasm. ${ }^{2,3}$ The 553 protein assists the determination of the DNA repair or the activation of programmed cell death (apoptosis), ${ }^{7}$ a characteristic of the negatively coordinated cell growth. Initiation of p53 protein may competently inhibit cell growth and signify cell death (apoptosis). Protein E6 of the transforming HPV (16/18) may efficaciously combine with protein $\mathrm{p} 53$. The viral protein E6 engages the cellular protein E6 AP which is employed as a Ubiquitin ligase for the p53 comprising complex. Ubiquitination of the p53 emerges due to the protein retention, followed by marked degradation. In the absence of $\mathrm{p} 53$ protein, the cell is deprived of the ability to discern and restore the latent, devastated DNA. Thus the cell division may persist without cellular rejuvenation. ${ }^{1,7}$ Remodelled, invariable protein $\mathrm{p} 53$ may be ascertained by immune-histochemistry. ${ }^{1}$

Analysis and attributes: Oral squamous papillomas are sui generis lesions. The preponderantly female, solitary lesions appear at a variable age-group. The lesions are frequently detected at the tongue, followed by the palate, lips, labial commissures and may emerge within 2 months to 20 years, following viral exposure. ${ }^{1,2}$ On morphology, the spherical lesions are commonest, followed by the cauliflower architecture. The magnitude of the lesions varies from 0.2 to 1.2 centimetre. The predominantly pedunculated lesions may be flaccid or soft with a gray/white hue. Solitary lesions generally reoccur infrequently. The clinical manifestations of the composite lesions are characteristic ${ }^{2}$ The benign oral lesions may demonstrate a concomitant viral aetiology with human papilloma virus (HPV),
Volume 6 Issue 4 - 2018

\author{
Anubha Bajaj \\ University Panjab University, India
}

Correspondence: Anubha Bajaj, Designation Consultant Pathologist, University Panjab University, Chandigarh, India, Tel +00911 I251 17399, 00919811693956,

Email anubha.bajaj@gmail.com, anubha.bajaj@yahoo.com

Received: June 14, 2018 | Published: July 30, 2018

specifically 2, 4, 6,11, 13, 32 sub-types. ${ }^{3,8}$ The malignant lesions are concordant with human papilloma virus (HPV) sub-types 16,18 , and 33 .

Viral duplication of the human papilloma virus appears in heterogeneous epithelial cells and the extrinsic epithelial lamina may be discarded in order to dispense with the viral particles. The antigenic stimulus is usually contained and so is the immune modulation and susceptibility to viral infection. Hence the Human Papilloma Virus (HPV) is interminably contagious and difficult to eradicate. ${ }^{3,6}$ Lesions may persist up-to 20years following infection, in the absence of significant metamorphosis. The virus may practically inhabit all the lesions, as evidenced on histopathology by the occurrence of koilocyte like cells in the spinous epithelial substratum. ${ }^{1,9}$ Basilar hyperplasia is infrequent and when accompanied with mitosis, it may be misinterpreted as mild epithelial dysplasia. However, the benign oral squamous papilloma may occasionally display atypical cells and mitosis. The characteristics may be suspicious of a Papillary Carcinoma. The compact, cornified, stratified epithelium illustrates a whitish chromaticity. The HPV-DNA in the host epithelium may be identified by a Polymerase chain reaction (PCR) and in situ hybridization (ISH) techniques, utilizing radioisotope labelled specific probes. ${ }^{1}$ The polymerase chain reaction (PCR) is an appropriate and innovative technique for the viral (HPV) delineation, in contrast to the in situ hybridization (ISH). The low-grade oncogenic variants of the human papilloma virus, HPV 6 and 11 are implicated in the genesis of Papillomas. ${ }^{6,10}$

Precancerous cells may convert into definitive malignant cells, aided by the dormant genes which govern the cell multiplication such as the $\mathrm{p} 53$ proteins. Genetic mutation of the $\mathrm{p} 53$ ameliorates the protein and augments the gene p53, ${ }^{11,12}$ diminishes the cellular containment , augments the cellular proliferation and consequent malignant transformation. However, malignant tumours of the oral cavity may not singularly be generated by the onco-genes. Multitudinous mechanisms inactivate the protein p53 and may be comprised of analogous viral or cellular onco-proteins. E6 of HPV 16 adapts to the protein binding, enhances protein degeneration and contains the cellular proliferation. ${ }^{8,13,14}$ Infection with HPV and/or metamorphoses of protein p53 may be synchronized in the squamous papillomas of the oral cavity. Intense nuclear immune-staining of protein p53 complies 
with the hypothesis that dual nuclear aggregation of mutant and wild type of protein $\mathrm{p} 53$ enhances the cellular growth.

Histopathological statutes: The squamous epithelium displays finger like projections, the epithelial maturation pattern is orderly with epithelial hyperkeratosis. Spinous epithelial cells may depict cytoplasmic vacuolation with pale, clear perinuclear haloes, nuclear pyknosis and infrequent basilar hyperplasia. Oral squamous papillomas frequently display koilocytosis on account of the coexistent cytopathic influence. Atypical nuclear alterations may be exemplified; particularly with immune-compromised (HIV positive) individuals. ${ }^{9}$ The epithelium displays a typical maturation pattern with prominent hyperkeratosis. Epithelial to basal cell quantification is moderate (basilar hyperplasia) along with an extensive koilocytic transition. ${ }^{1}$ Virally induced carcinomas are the poorly differentiated, non-keratinizing tumours and may be enunciated in the tonsils of

\begin{tabular}{lc}
\hline Oral disease & $\begin{array}{c}\text { human papilloma } \\
\text { virus types }\end{array}$ \\
Verruca vulgaris & 2,4 \\
Condyloma lata & 6,11 \\
Squamous papilloma & 6,11 \\
Focal epithelial hyperplasia & 13,32 \\
Oral leukoplakia & 16,18 \\
Verrucous carcinoma & $6,11,16$ \\
Oral squamous-cell carcinoma & 16,18 \\
Laryngeal papilloma & $6,11,30$ \\
Maxillary sinus papilloma & 57 \\
\hline
\end{tabular}

Figure I Viral Imputations in miscellaneous squamous lesions.

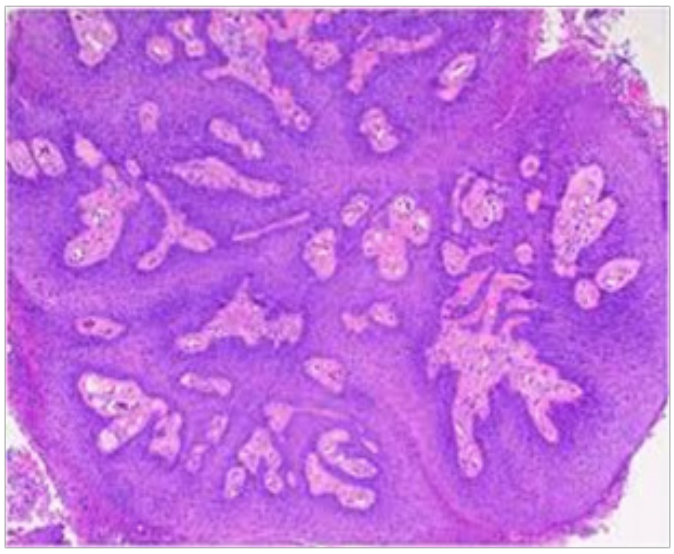

Figure 3 Papillomas with a fibro-vascular core.

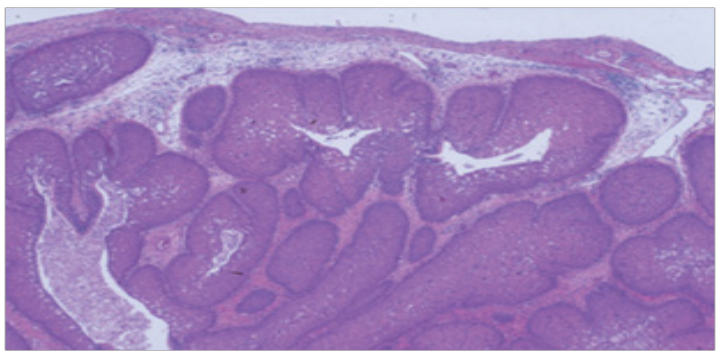

Figure 5 Inverted Papilloma with epithelial hyperplasia. sexually active young men. Majority of the tumours elucidate the p16 proteins. Oro-pharyngeal cancers which are reactive for human papilloma virus (HPV) frequently depict basaloid morphology. Apart from a viral genesis, squamous papillomas may ensue from mechanical irritation or may be genetically determined as a constituent of Cowden's syndrome.

Immunohistochemical assay: Antibodies BP53-12 and PAb 240 may be elucidated with the $\mathrm{p} 53$ proteins and require evaluation. BP53-12 determines the mutant and wild type of p53 whereas the PAb240 is mutant specific. Immune-markers BP53-12 and PAb 240 may be exemplified in the entire epithelial thickness (basal, spinous, granular and cornified cell layers), estimated for the appearance of p53 proteins and may be discerned as non-reactive or weakly reactive.,11 (Figures 1-11).

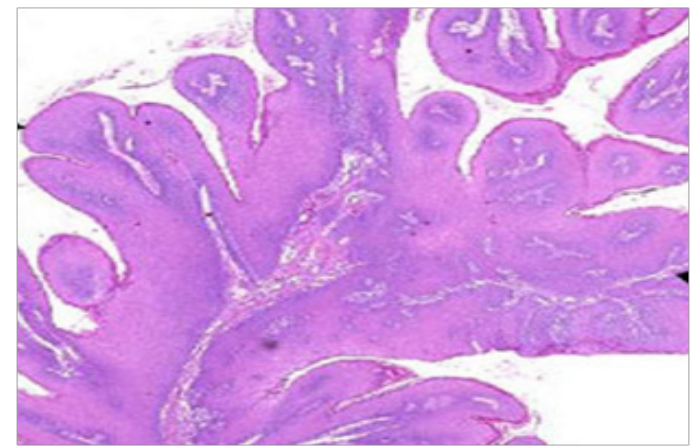

Figure 2 Oral Squamous Papilloma with papillary projections.

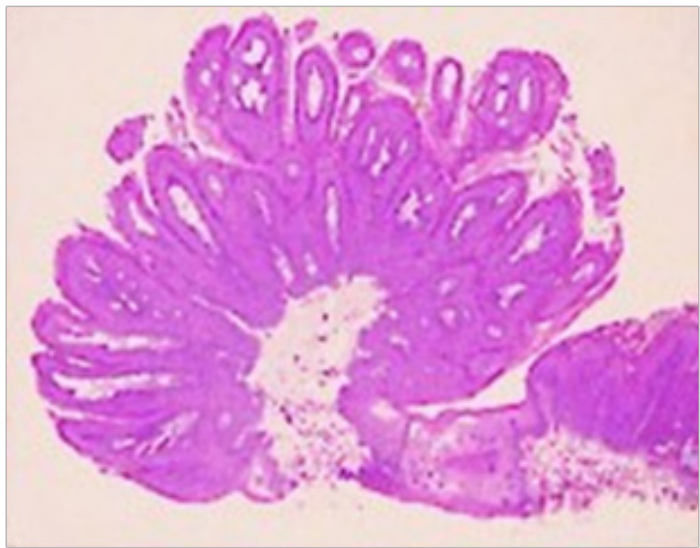

Figure 4 Papillomatous lesions with a prominent fibro-vascular core.

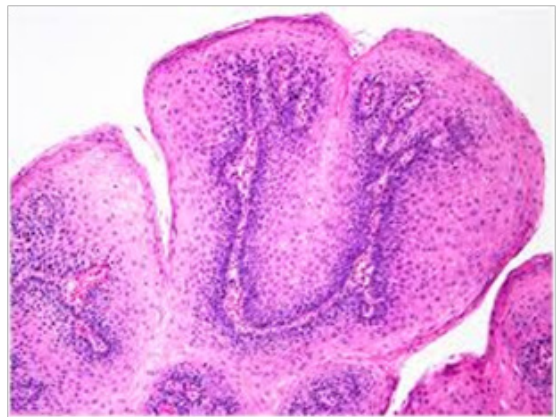

Figure 6 Squamous papilloma- oesophagus. 


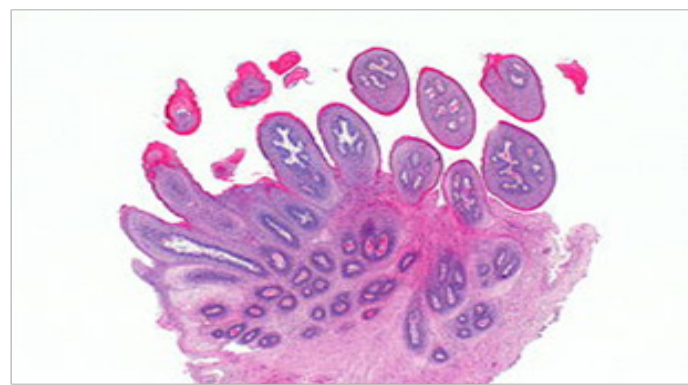

Figure 7 Papillomas with buried epithelial glands.

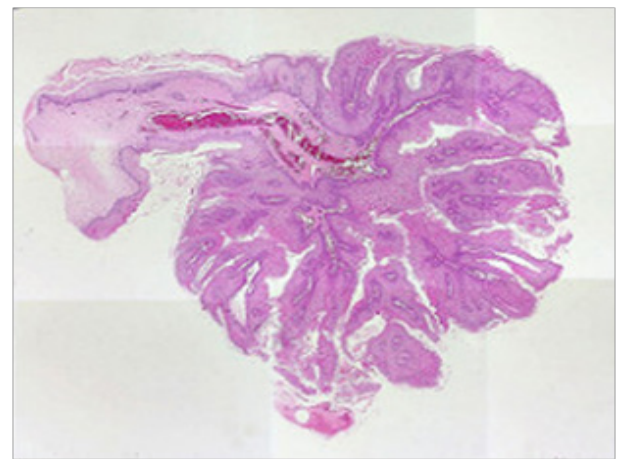

Figure 9 Squamous epithelium lined Papillomas-Uvula.

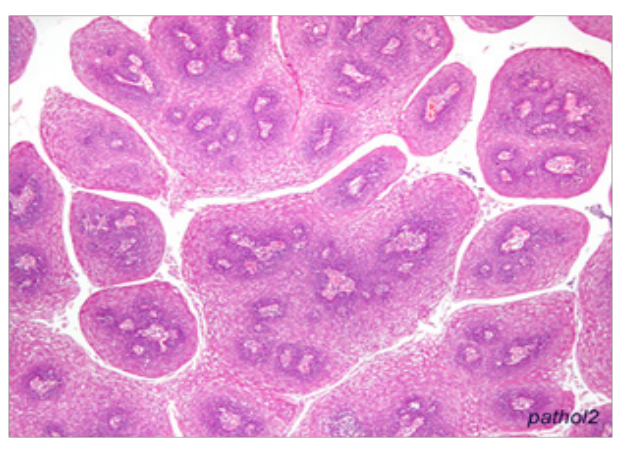

Figure 8 Multiple papillomas-pharynx

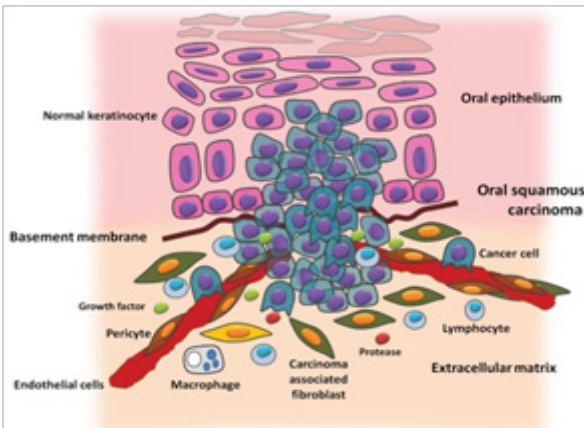

Figure 10 Tumour micro-environment.

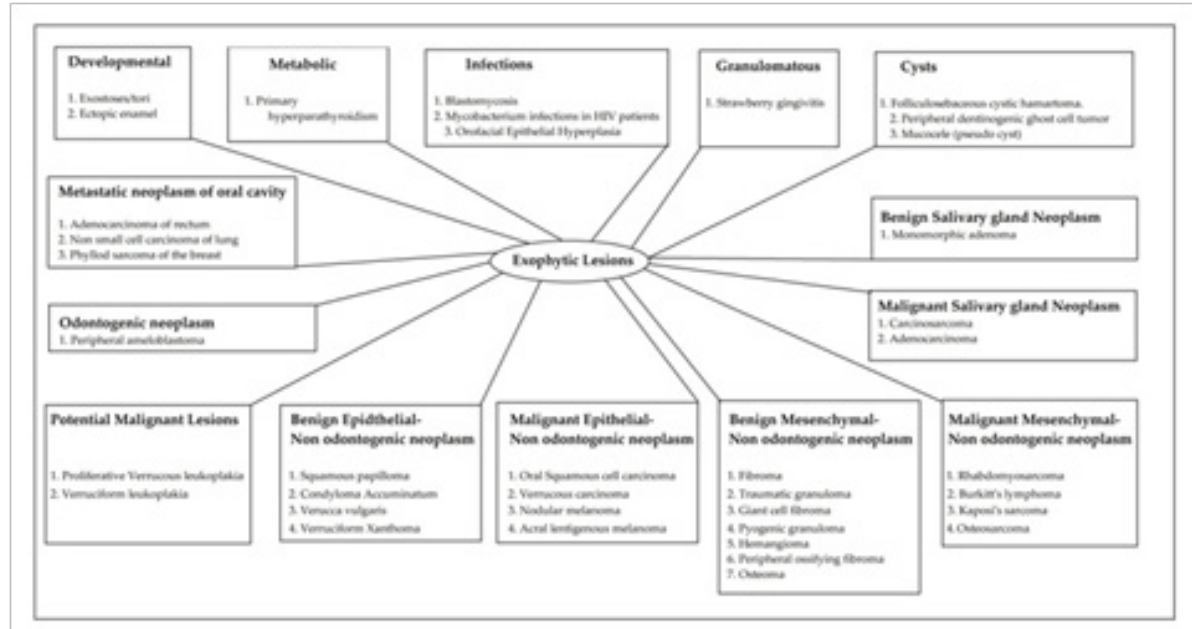

Figure I I Adjudication of oral exophytic lesions.

Molecular biomarkers: Sustainable and paramount markers in the oral squamous cell carcinoma (OSCC) appearance and progression are the Epidermal growth factor receptor (EGFR), Human Papilloma Virus (HPV), Transforming Growth Factor $\beta$ (TGF $\beta$ ), EpithelialMesenchymal transition(EMT) and the markers of hypoxia. The specific lineage bio-markers for numerous sub-categories are SOX-2, TP-63 CyclinD1 (CDND-1), PIK 3 CA (Phosphotidyl inositol 4,5biphosphate 3 kinase, catalytic subunit alpha iso form, the EGFR oncogene including KEAP1/NFE2L2(Kelch like ECH associated protein1)/Nuclear Erythroid-2 related factor 2) and the dys-regulation of the Oxidative stress pathway $(3,4)$.

i. Genetic and epigenetic conversion in oral squamous cell carcinoma (OSCC) may elucidate a decline or over-expression of proteins. Cyclin D1, p53, retinoblastoma, Epidermal Growth
Factor Receptor(EGFR), Signal transducer and Activator of Transcription 3, Vascular Endothelial Growth Factor (VEGF) receiver and adjunctive molecules may be extremely modified. ${ }^{15,16}$

ii. Ki-67 and p53 frequently manifest cellular proliferation. p53 is a transcription factor which is implicated in cell cycle control, apoptosis and maintenance of genetic stability. Mutation of the $\mathrm{p} 53$ gene may be encountered in more than half the cases $(>50 \%)$ of oral squamous cell carcinoma (OSCC). p 53 may be engaged on account of the injury to the DNA, hypoxia and oncogene activation. Ki-67 is usually elucidated in the phases of cell cycle G1, S, G2 and M. Tumour relapse and patient survival may be accurately conjectured with the assessment of Ki $67 .^{15,16}$ 
iii. Homeo-box (HOX) genes: Over expression of the specific markers is associated with carcinogenesis, particularly head and neck neoplasm. ${ }^{15,16} \mathrm{HOX}$ B7 belongs to the family of the homeodomain transcription factors and regulates the development, proliferation and survival of progenitor cells. HOX B7 is usually over expressed in oral squamous cell carcinoma (OSCC) and may delineate a harmful prognosis. ${ }^{15,16}$

iv. Collagen type IV (Coll IV) is a valuable protein component of the basement membrane. The integrity of the membrane may be transformed via the matrix metallo-proteinases (MMP2 \& MMP 9) as demonstrated in the oral squamous cell carcinoma (OSCC) and the abutting tissues. Thus supervising the manifestation of the Coll IV may decree a prognostic significance. ${ }^{15,16}$

v. Tumour Micro-environment (TME): Is constituted of a variable cellular component of fibroblasts, cancer associated fibroblasts(CAFs), myo-fibroblasts, smooth muscle cells, endothelial cells with precursors, pericytes, neutrophils, eosinophils, basophils, mast cells, T \& B small lymphocytes, natural killer (NK) cells, antigen presenting cells such as macrophages and dendritic cells. ${ }^{15}$

vi. Cancer Associated Fibroblasts (CAFs): are frequent elements of the tumour micro-environment (TME). CAFs are reactive for smooth muscle actin CAFs may be predominantly situated at the tumour perimeter. CAFs may be augmented in the oral squamous cell carcinoma (OSCC) and is a manifestation of tumour infiltration (Table 1). ${ }^{17}$ It modulates the microenvironment for tumour invasion and distant metastasis and indicates a poor prognosis. $^{15}$

Table I Sub-Categories of Oral Squamous Cell Carcinoma. I7

$\begin{array}{lll}\text { Class 1 } & \begin{array}{l}\text { Human } \\ \text { Papilloma Virus } \\ \text { class. }\end{array} & \begin{array}{l}\text { Up-regulation of gene, HPV association, } \\ \text { cell proliferation. Over-expression of } \\ \text { EGFR,TGF- } \beta, \text { RAS, Cyclin D1. }\end{array}\end{array}$

\section{Class 2 Mesenchymal and angiogenesis, WNT and onco-signature.} Over-expression of EGFR, TGF- $\beta$, RAS, Cyclin D1.

\begin{tabular}{|c|c|c|}
\hline Class3 & $\begin{array}{l}\text { Hypoxia } \\
\text { accompanied }\end{array}$ & $\begin{array}{l}\text { Hypoxia, drug metabolism pathway, } \beta \\
\text { catenin pathway and biotic response. }\end{array}$ \\
\hline Class 4 & $\begin{array}{l}\text { Defence } \\
\text { response/ } \\
\text { Inflammatory }\end{array}$ & $\begin{array}{l}\text { Enriched interferon response pathway and } \\
\text { gene associated with ALK-onco-signature. }\end{array}$ \\
\hline Class 5 & Classical & $\begin{array}{l}\text { Activation of protein kinase } \mathrm{B}(\mathrm{AKT}) \text { and } \\
\text { xenobiotic pathway(Aldo-keto-reductase } \\
\text { family member } \mathrm{C} 1 / 3 \text {. AKR C } 1 / 3 \text { and } \\
\text { NFE2L2). }\end{array}$ \\
\hline Class6 & Immune-reactive & $\begin{array}{l}\text { Up-regulated immune related pathway and } \\
\text { Interferon (IFN). }\end{array}$ \\
\hline
\end{tabular}

Group I (Basal Tumour Like): Delineates a maximal expression of $\mathrm{P}$ cadherin, Laminin2, BPA -1 bullous pemphigoid antigen-1), Kallikrein 10, Collagen XVII . Adjunctive genes depicted are Desmocollin 2, Desmoglein3, Cytokeratin 14, Transforming Growth Factor $-\beta$ (TGF $\beta$ ), Fibroblastic growth factor (FGF-BP) and MMK 6 (a mitogen activated protein MAP kinase) which are important activators of the Epidermal Growth Factor Receptor (EGFR) pathways (EGFR- pathway signature) $(4,6)$.
Group 2 (Mesenchymal enriched subype): Chiefly elucidates Vimentin, Syndecan, Lysyl-Oxidase and Collagen.

Group 3 (Normal epithelium type): Genes for microsomal Glutathione S Transferase 2, Cytokeratin 15, Cytokeratin 4 and Cytokeratin 14 may be demonstrated.

Group 4 (Anti oxidant): An amplification of the antioxidant induced enzymes involved in xenobactic metabolism which incorporates the Glutathione-S-Transferase M3 (GSTH 3), thioredoxin reductase, and Glutathione peri-oxidase 2, Aldo keto reductase 1 and genes of the pentose phosphate cycle may be enunciated. ${ }^{18}$

\section{Conclusion}

A benign proliferation of the stratified squamous epithelium with a papillary or verrucous exophytic growth is defined as the Oral Squamous Papilloma. A viral infectious aetiology with Human Papilloma Virus (HPV) and a 150 varieties of virus have been indicated in the genesis of the lesion. ${ }^{16}$ The benign oral lesions may demonstrate a concomitant viral aetiology with human papilloma virus (HPV) specifically 2, 4, 6,11, 13, 32 sub-types. The malignant lesions are concordant with human papilloma virus (HPV) sub-types 16, 18, and 33. The HPV-DNA in the host epithelium may be identified by a Polymerase chain reaction (PCR) and in situ hybridization (ISH) techniques, utilizing radioisotope labelled specific probes. Immunereactive assays for p53 protein are generally negative or weakly positive, thus conferring the typically benign lesion with a probability of malignant conversion. Immune-markers BP53-12 and PAb 240 may be exemplified in the entire epithelial thickness (basal, spinous, granular and cornified cell layers). The specific lineage bio-markers for numerous sub-categories of the oral squamous cell carcinoma(OSCC) are SOX -2, TP-63 CyclinD1 (CDND-1), PIK 3 CA (Phosphotidyl inositol 4,5- biphosphate 3 kinase, catalytic subunit alpha iso form, the EGFR oncogene including KEAP1/NFE2L2 (Kelch like ECH associated protein1)/Nuclear Erythroid-2 related factor 2) and the dys-regulation of the Oxidative stress pathway. ${ }^{19-33}$

Group I maximally expresses P cadherin, Laminin2, BPA -1 (bullous pemphigoid antigen -1), Kallikrein 10, Collagen XVII a. Adjunctive genes depicted are Desmocollin 2, Desmoglein 3, Cytokeratin 14, Transforming Growth Factor $-\beta$ (TGF $\beta$ ), Fibroblastic growth factor (FGF-BP) and MMK 6 (a mitogen activated protein MAP kinase). Group 2 chiefly elucidates Vimentin, Syndecan, LysylOxidase and Collagen. Group 3 may depict genes for microsomal Glutathione S Transferase 2, Cytokeratin 15, Cytokeratin 4 and Cytokeratin 14. Group 4 amplifies the antioxidant induced enzymes involved in xenobactic metabolism which incorporates the Glutathione-S-Transferase M3 (GSTH 3), thioredoxin reductase, and Glutathione peri-oxidase 2, Aldo keto reductase 1 and genes of the pentose phosphate cycle.

Class 1 oral squamous cell carcinoma (OSCC-human papilloma virus subtype) respond favourably to diverse therapeutic approaches whereas Class 2 (mesenchymal subtype) and Class 3 oral squamous cell carcinoma (OSCC -hypoxic subtype) demonstrate an unfavourable outcome.

\section{Acknowledgements}

None. 


\section{Conflict of interest}

The author declares that there is none of the conflicts.

\section{References}

1. Thalassa E Carneiro, Marinho SA, Verli FD, et al. Oral Squamous Papilloma :clinical, histologic and immunohistochemical analyses. Journal of Oral Science. 2009;51(3):367-372.

2. Abbey LM. The clinical and histopathological features of a series of 464 oral squamous papillomas. Oral Surg Oral Med Oral Pathol. 1980:49(5):419-428.

3. Yamaguchi T, Shindoh M, Amemiya A, et al. Detection of Human Papilloma Virus type 2 related sequence in oral papilloma. Anal Cell Pathol. 1998:16(3):125 -150.

4. Neville BW. Oral Maxillofacial Pathology. 2nd ed. 2008. p. 984.

5. Kumar V. Robbins and Cotran-Pathologic Basis of Disease. 9th ed. Canada: Rio de Janeiro; 2005:357-432.

6. Burd EM. Human Papilloma Virus and Cervical Cancer. Clinical Microbiol Rev. 2003;16(1):1-17.

7. Black AP. The role of p53 in the immunobiology of squamous cell carcinoma. Clin Exp Immunol. 2003;132(3):379-384.

8. Jiminez C, Correnti M, Salma N, et al. Detection of Human Papilloma Virus DNA in benign oral squamous epithelial lesions in Venezuela. $J$ Oral Pathol Med. 2001;30(7):385-388.

9. Rosai J. Rosai and Ackerman's Surgical Pathology. 10th ed. 2011; p 2892.

10. Eliashar R. A case of squamous papilloma after uvulopalatopharyngeoplasty. Ear, Nose and Throat J .2000;79(4):250 251.

11. Chong KT. High level of expression of human epithelial $\beta$ defensins (hBD 1-3) in Papillomavirus induced lesions. Virol J. 2006;3:75.

12. Chiba I, Shindoh M, Yasuda M. Mutations in the p53 gene and Human Papilloma Virus infection as a significant prognostic factor in Squamous Cell Carcinoma of the oral cavity. Oncogene. 1996;12(8):1663-1668.

13. Mao EJ, Stephen M Schwartz, Janet R Daling, et al. Human Papilloma Virus and p 53 mutations in normal premalignant and malignant oral epithelia. Int J Cancer. 1996:69(2):152-158.

14. Badaracco G, Aldo Venuti, Armando Bartolazzi, et al. Over-expression of P53 and BCL 2 protein and presence of HPV infection are independent events in head and neck cancer. J Oral Pathol Med. 2000;29(4):173-179.

15. Cesar Rivera. Histological and molecular aspects of oral squamous cell carcinoma (review). Oncol Lett. 2014;8(1):7-11.

16. Radhika Manoj Baule, Venugopal R, Konda P, et al. Molecular classification of Oral Squamous Cell Carcinoma. J Clin Diagn Res. 2016;10(9):ZE18-ZE-21.

17. Cecco LD, Nicolau M, Giannoccaro M, et al. Head and Neck CancerSubtypes with Biological and Clinical Relevance- Meta -analysis of gene expression data. Oncotarget. 2015:6(11):9627-42.

18. Chung HC, Parker JS, Karaca G, et al. Molecular classification of head and neck squamous cell carcinoma using pattern of gene expressions. Cancer Cell. 2004;5(5):489-500.

19. Walter V, Xiaoying Yin, Matthew D. Wilkerson et al. Molecular subtypes in head and neck cancer exhibit distinct patterns of chromosomal gain and loss of canonical cancer genes. Plos One. 2013:8(2):e56823.

20. Silva AMTC, Cruz ADD, Silva CCD, et al. Genotyping of Human Papilloma Virus in patients with recurrent laryngeal papillomatosis. Rev Bras Cancerol. 2003:49(3):167-174.

21. Gonzalez ML, Pineda Ramírez L, Pineda Ramírez L, et al. Papiloma Bucal-estudio retrospective de 1963 a 1982. Rev Med. 1986;24(2):117120.

22. Halazonetis TD, Davis LJ, Kandil AN. Wild type p53 adopts mutant like conformation when bound to the DNA. EMBOJ. 1993;12(3):10211028.

23. Image 1 Courtesy: Journal of Cancer Research and Therapeutics.

24. Image 2 Courtesy: Med-aid India.com.

25. Image 3 Courtesy: Pathology Outlines.

26. Image 4 Courtesy: MRCO phth.com.

27. Image 5 Courtesy: Internet Scientific Publications.

28. Image 6 Courtesy: Journals elseiver health.

29. Image 7 Courtesy: Wikipedia.com

30. Image 8 Courtesy: Photo-library of pathology.

31. Image 9 Courtesy: Five prime.

32. Image 10 Courtesy: Spandidos publications.

33. Image 11 Courtesy: Journal of Clinical \& Diagnostic Research. 